\title{
Photo Skin Rejuvenation with IPL
}

\author{
Aristides Arellano Huacuja*, Dafne ArellanoMontalvo, Anja Arellano Montalvo \\ Dermatological Clinic and Aesthetic Surgery of Puebla. Puebla, Mexico.
}

Received: July 05, 2021; Accepted: July 15, 2021; Published: August 01, 2021

*Corresponding author: Arístides Arellano Huacuja, PlasticSurgery, Clinic of Dermatology and AestheticSurgery of Puebla, Puebla, Pué, Tel. No: (222)437740, Fax: (222)2377494; E-mail: aristidesarella@yahoo.com.mx

\section{Introduction}

Photo aging is becoming increasingly interesting, as it causes, among other alterations, the appearance of wrinkles, pigmentation disorders and loss of normal skin tone. Exposure to sunlight is responsible for 80 to $90 \%$ of facial aging. Signs of photoaged skin include changes in skin color, dyschromia's, lentigos, gradual appearance of telangiectasias (dilation of small blood vessels), as well as changes in skin texture such as loss of smoothness, keratotic appearance, early development and deepening of wrinkles.The aging of our skin is not only the result of the passage of time. It is also strongly influenced by external factors, such as weather conditions. The skin is made up of three layers: the epidermis, or outer layer, the dermis, or middle layer, and the subcutaneous tissue, or deep layer. The dermis contains collagen, elastin, and other fibers that make up the skin's structure. These elements give the skin a smooth and youthful appearance, and these are those damaged by Ultraviolet Radiation (UVR). UVRs are made up of two different types of waves, UVA and UVB.

When UV rays hit the skin, the melanocytes struggle to produce melanin to deposit in the epidermis. This is the process that gives you a tan, which is actually a defense reaction against radiation so that it does not penetrate the skin. UVB rays are shorter than UVA rays and are the main cause of sunburn. UVA rays are responsible for much of the damage we associate with photoaging. UVA rays penetrate deep into the dermis, where damage to collagen fibers occurs. This damage causes an abnormal increase in elastin production.

The unusual amounts of elastininduce the production of enzymes called metalloproteinases. These enzymes, which rebuild damaged collagen, work incorrectly and degrade it, causing the skin to rebuild itself incorrectly. As this process is repeated with daily UVA exposure, improperly rebuilt skin forms wrinkles, and the reduction in collagen results in atrophic, aged-looking skin.Continuous exposure to the sun can also cause staining. A brownish age spot is actually a lentigo, a pigmentation caused by sun exposure. Age spots generally appear on the hands, arms, face, and back in men and women.

The best way to fight photoaging is through prevention. The daily application of a sunscreen with SPF of 15 or more to the areas vulnerable to photoaging, not only helps prevent it, but can also reverse some of the existing signs. Reducing exposure to UV radiation lowers the risk of developing skin cancer or a precancerous lesion.

Photo rejuvenation is a treatment that applies intense light to stimulate skin regeneration and collagen formation.In addition to significantly improving the appearance of our skin, it also manages to eliminate damaged skin and provide a rejuvenated appearance. Thanks to Photo rejuvenation, the expectations of patients have been increasing since they can combat their skin problems. The objective of this treatment is to achieve a high rate of cosmetic benefit in the minimum number of sessions and with a short recovery time. The three elements on which we can work are the support, the texture and the pigmentary alterations of the skin.

Light systems such as the one we use in photo rejuvenation allow us to combine different levels of treatment and constitute a complement to surgical procedures, providing a "biological" concept by stimulating regeneration and neo-collagen genesis. The clinical indications in the field of Dermatology and Plastic Surgery include skin rejuvenation as an effective alternative for the treatment of pigmentation, loss of skin tone, some vascular malformations, the elimination of telangiectasias, which are the result of photo aging. in most cases premature.

\section{What is pulsed light equipment?}

Pulsed light equipment is a device that is composed of an active medium, in this case solid, enclosed inside a resonant cavity limited by a pair of flat and parallel mirrors, when most of the atoms or molecules are excited and elevated. At a stable energy level, an amplification of light occurs inside, part of this light escapes to the outside through the semi-transparent mirror in the form of pulses.

To achieve an action on a certain element, this light energy must be absorbed by the components of that element. The chromiumrelated elements (which absorb light energy in the skin) are hemoglobin in the dermal vessels and melanin in the skin. Therefore, if an element does not absorb this energy in the form of light, there would be no changes in it; We call this characteristic the principle of selectivity. The light energy can be directed towards the substance or tissue that absorbs it without affecting the surrounding tissues that do not absorb it.

This tool based on selective photo thermolysis has allowed expanding the field of its clinical applications, thanks to the fact that it emits wavelengths between 550 and 1,200 nm, non- 
ablative photo rejuvenation of the skin is considered one of the innovative techniques in the field. Of cosmetic dermo as it offers multiple advantages: it allows the global treatment of the entire affected skin surface (face, neck, décolleté, back of hands), not requires a recovery or convalescence period, is indicated at any age, is repeatable and can be combined with other methods such as implants, fillers or botulinum toxin. The doctor will make an assessment of the patient's skin type photo, based on that and the type of injury that he presents, the energy parameters that will be useful to improve his problem will be chosen

\section{Materials and Methods}

Both the person performing the procedure and the patient wear goggles to prevent eye damage. Next, a thin layer of gel is placed and over it the head of the equipment in the area to be treated (face, neck, forearm, hands, etc.), all this having been selected the filter and the appropriate power for each type of treatment and skin.

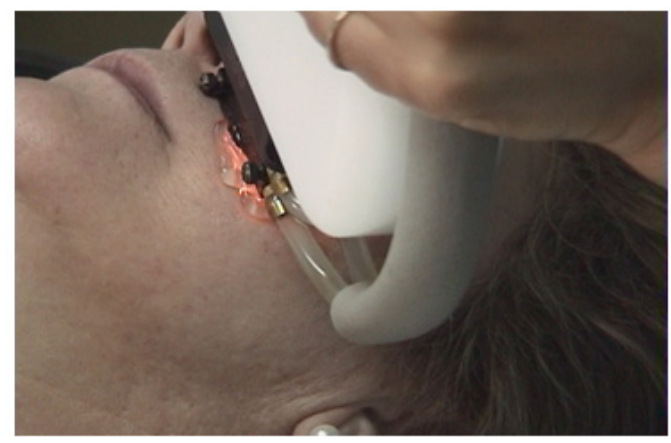

The pulsed light works by heating the skin through the application of intense light pulses, which will penetrate the skin, stimulating the fibroblasts to form collagen. If the IPL used is soft, only the first layer of skin will be eliminated and the greater the intensity of the IPL used, the deeper layers can be reached.

Once the light is applied to the area to be treated, it begins to act through heat, directly attacking the melanin of the pigmented spot, in this way the cells begin to be eliminated and to be replaced by new cells.

This treatment does not need too many sessions, although it will depend on the objective and the area to be treated, generally 6 sessions are carried out that last approximately 45 - 60 minutes each. Between one session and another, it is convenient to allow 3 to 4 weeks to pass. In addition, it has the benefit of being a painless outpatient treatment that does not require recovery time.By causing cell regeneration, it is likely that at the end of the treatment a slight redness or slight temporary inflammation will appear, which will disappear over the days.

Although it is a treatment that can be performed on any patient, it is advisable not to tan for the duration of the treatment and to use sunscreen to protect the skin.

\section{Results}

The results obtained in most cases during 20 years of carrying out this treatment in various pathologies such as Poikiloderma, telangiectasias, café-au-lait nevus, Becker nevus, Hyper pigmentation, among others, have been highly satisfactory.It must be considered environmental factors, uses and customs of the population and important racial characteristics in our environment.

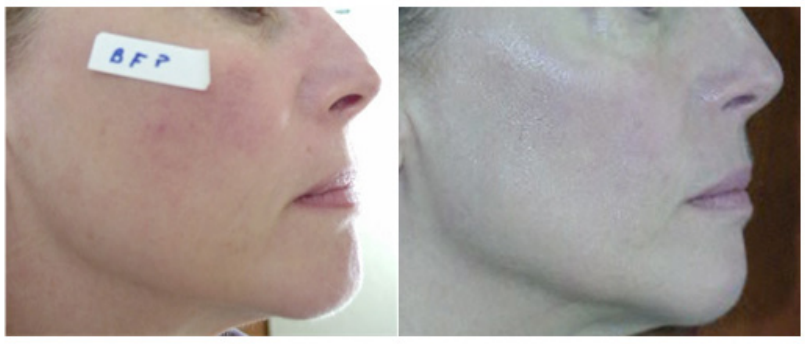

Before and after Telangiectasia IPL treatment

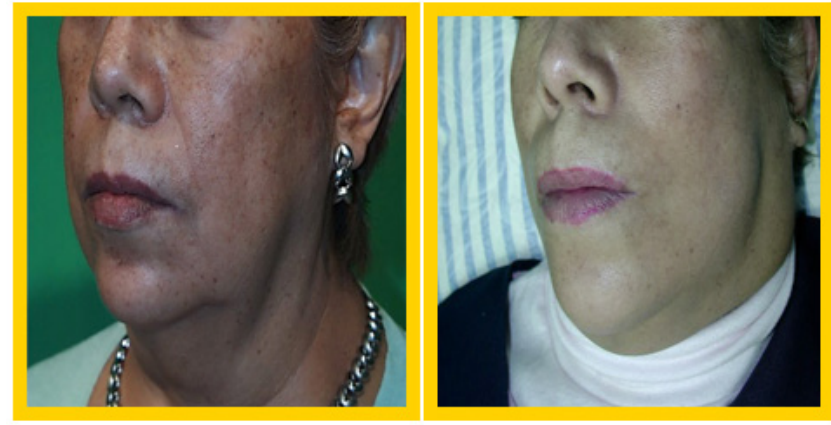

Before and after hyper pigmentation IPL treatment

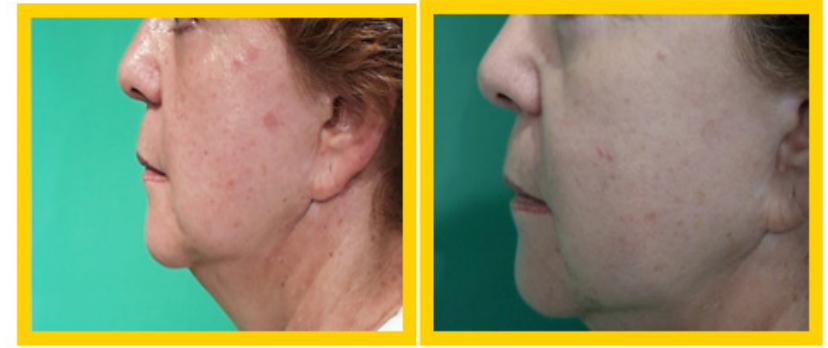

Before and after Lentigos IPL treatment

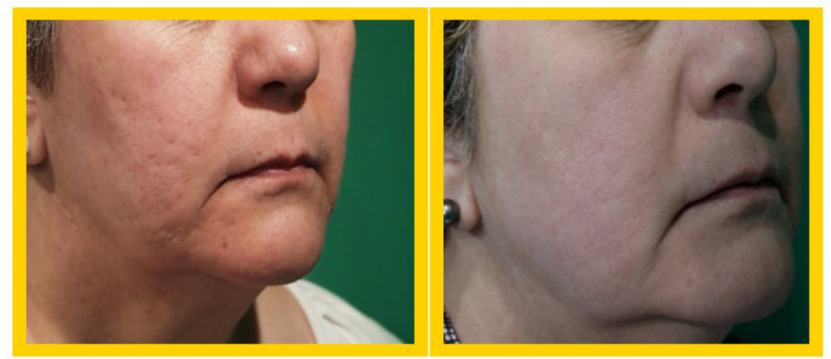

Before and after Acne scars IPL treatment 


\section{Conclusion}

We have treated more than 10,756 patients with this technology allowing a clearance of the pathology that affected them between 80 to $90 \%$ in most cases.

The treatment is easy to carry out for both the patient and the treating physician, but the perseverance and care of the patient makes the result as expected, since as there are multiple sessions, the abandonment of this is frequent.

\section{References}

1. Santofimia R, Olaso SA. Laser, pulsed light, radiofrequency and other sources of energy. Cir plást iberolatinoam. 2008;34:61-70

2. Armijo M, Camacho F. Treaty of Dermatology. Group Aula Medica Spain. 1998;1:362-375.

3. Bjerring $\mathrm{P}$, Christiansen $\mathrm{K}$. Intense pulsed light source for treatment of small melanocytic nevi and solar lentigines. J Cutan Laser Ther 2000;2(4):177-181.

4. Cisneros VJL, Camacho Martinez F. Laser sources of intense pulsed light in dermatology and dermocosmetics. Mold. 2002.

5. Fitzpatrick T, Eisen, Wolf, Freedberg, Austen. Dermatology in General Medicine. Panamericana. 1993

6. Pessa JE. An algorithm of facial aging: verification of Lambros's theory by three dimensional stereolithography, with reference to the pathogenesis of midface aging, sclera show, and the lateral suborbital through deformity. Plast Reconstr Surg. 2000;106(2):479-488.

7. Utley DS, Goode RL. Radiofrequency ablation of nerve to the corrugator muscle for eliminated of glabellar furrowing. Arch Facial Plas Surg. 1999;1(1):46-48
8. Seckel BR, Kovancha CJ, Cetrulo CL, Passmore AK, Meneses PG, White T. Laser blephoroplasty with transconjuntival orbicularis muscle septum tightening and periocular skin resurfacing: a safe and advantageous techniques. Plast Reconstr Surg. 2000;106(5):1127-1141.

9. Field LM. Cascading epicanthol skin resection and aesthetic reconstruction. Dermatol Surg. 2000;26(9):888-889.

10. Guerrissi JO. Surgical treatments of the senile upper up. Plast Reconstr Surg. 2000;106(4):953-954.

11. Gryskiewick JM. Alloderm lip augmentation. Plast Reconstr Surg. 2000;106(4):953-954.

12. Behroozan DS, Chistian MM, Moy RL. Short pulse carbon dioxide laser resurfacing of the neck. J Am Acad Dermatol 2000;43(1 pt 1):72-76.

13. Jasin ME. Regarding combined therapy for neck rejuvenation. Dermatol Surg. 2000;26(3):294-295.

14. Souza EB, Eraso PJ, Matsuda CA, Regozzini DU, Burgos DS, Acosta HA. Brachioplasty technique with the use of molds. Plast Reconstr Surg. 2000;105(5):1854-1860.

15. Wendt JR. Distal, dorsal superior extremity plasty. Plast Reconstr Surg. 2000;106(1):210-213.

16. Niamtu J. Cosmetic oral and maxillafacial surgery options. J Am Dent Assoc. 2000;131(6):756-764.

17. Gorman C. Face lift in a jar? Time. 2000;156(7):48-52.

18. Alster TS, West TB. Human-derived and new synthetic injectable materials for soft-tissue augmentation: current status and role in cosmetic surgery. Plast Reconstr Surg. 2000;105(7):2515-2525.

19. Sagi A. Regarding liposuction in patients over the age of 40. Dermatol Surg. 2000;26(9):901. 